\title{
PLAN DE ORDENAMIENTO TERRITORIAL. PLAN ESTRATÉGICO DE DESARROLLO URBANO TERRITORIAL, PRESIDENCIA DE LA PLAZA, CHACO, ARGENTINA
}

STRATEGIC PLAN OF TERRITORIAL URBAN DEVELOPMENT, PRESIDENCY OF THE PLAZA, CHACO, ARGENTINA

ALBERTO PATRICIO MAHAVE Facultad de Arquitectura y Urbanismo - UNNE

MOIRA CARRIÓ

Facultad de Ciencias Económicas - UNNE

NATALIA ALBENDAÑO

Facultad de Arquitectura y Urbanismo - UNNE

ARGENTINA

Se puede vivir dos meses sin comida y dos semanas sin agua, pero sólo se puede vivir unos minutos sin aire. La tierra no es una herencia de nuestros padres, sino un préstamo de nuestros hijos. El amor es la fuerza más grande del universo, y si en el planeta hay un caos medioambiental es también porque falta amor por él. Hay suficiente en el mundo para cubrir las necesidades de todos los hombres, pero no para satisfacer su codicia. Mahatma Gandhi. 


\section{RESUMEN}

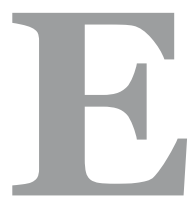

1 Plan Estratégico Territorial para la Municipalidad de Presidencia de la Plaza pone de manifiesto varios puntos claves, esenciales para el desarrollo equilibrado de las potencialidades y oportunidades dentro del entorno tanto local como externo.

La dimensión ambiental incluye temas como la calidad del aire y el agua, la adaptación al cambio climático, la reducción de la vulnerabilidad a las amenazas naturales y la cabertura de los servicios públicos; la dimensión del desarrollo urbano considera los aspectos físicos, económicos y sociales; y la dimensión legal aborda las características de una buena gobernabilidad, entre ellas la transparencia, participación pública y gestión pública moderna. Al mismo tiempo, examina los instrumentos normativos ya que son necesarios para el normal deselvonviemiento de la institución municipal y el desarrollo y crecimiento económico, social, urbano y territorial del municipio. Por ello, se pretende atender cuestiones que hacen al ordenamiento urbano, al espacio público y al medio natural, elementos estructurantes para el buen desarrollo del municipio tanto en lo social como en lo económico, con el objeto de producir acciones tendientes al funcionamiento óptimo del territorio y a la vez sustentable, garantizando a la población un ambiente saludable e integral. El éxito del Plan Estratégico Territorial para la Municipalidad de Presidencia de la Plaza depende de la voluntad política de las autoridades locales como de la ciudadanía, ya que resulta de vital importancia la sostenibilidad de las propuestas e intervenciones en corto, mediano y largo plazo que contribuirán a una modificación adecuada al territorio.

Palabras clave: planificación, ciudad, sostenible, equilibrio, identidad territorial.

\section{ABSTRACT}

The Strategic Territorial Plan for the Municipality of Presidency of the Square reveals several key points essential for the balanced development of the potentials and opportunities in both local and external environment. The environmental dimension includes topics as the quality of the air and the water, the adjustment to the climate change, the reduction of the vulnerability to the natural threats, and the tightening of the public services; the dimension of the urban development considers the physical, econom-ic and social aspects; and the legal dimension approaches the characteristics of a good governance, between them the transparency, public participation and public modern management. At the same time, it examines the normative instruments since they are necessary for the normal of the municipal institution and development and the economic, social, urban and territorial growth of the municipality. For it, one tries to attend to questions that they do to the urban classification, to the public space and the natural way, structuring elements for the good development of the municipality both in the social thing 
and in the economic thing, in order to produce actions tending to the ideal functioning of the territory and simultaneously sustainably, guaranteeing to the population a healthy and integral environment. The success of the Strategic Territorial Plan for the Municipality of Presidency of the Square depends on the political will of the local authorities as on the citizenship, since there ensues from vital importance the sustainability of the offers and interventions in short, medium and long term that will contribute to a proper modification to the territory.

Keywords: planning, city, sustainable, balance, territorial identity.

\section{1.- INTRODUCCIÓN}

\section{1 | METODOLOGíA}

El presente Plan fue formulado como iniciativa de la Dirección Nacional de Preinversión Municipal, DINAPREM, y posteriormente del Municipio de Presidencia de la Plaza, en materia de fomentar políticas urbanas estableciendo modalidades de planificación, desarrollo y gestión integrada y responsable, con el fin de corregir problemas que presenta el departamento y el municipio en la actualidad y el futuro.

El mismo es una herramienta concreta para la recuperación del territorio que mediante una sucesión de lineamientos, pueda alcanzar la construcción de un territorio integrado y equilibrado en todas sus direcciones. Esto implica analizar y reflexionar sobre las problemáticas de crecimiento, movilidad, infraestructura, preservación, valorización y rehabilitación del patrimonio, como así también tener presente el crecimiento de la población, para poder luego de un diagnóstico elaborar propuestas tendientes a posibilitar el acceso a suelo urbanizado de manera equilibrada con la inclusión de todos los actores que en su conjunto conforman la ciudad.

Se pretende abordar asimismo, la problemática a través de cuatro dimensiones vinculadas (Dimensión Urbana, Socio-Económica, Ambiental y Legal).

Por ello, esta estratégica iniciativa origina oportunidades para canalizar la planificación y ordenamiento del municipio, posibilitando y fortaleciendo el desarrollo de innovadoras alternativas para llevar adelante con total eficacia y eficiencia las acciones a implementar.

En una instancia analítica, se planteó un enfoque teórico sistemático para encarar la problemática territorial que presenta actualmente el departamento Presidencia de la Plaza, que a partir del análisis de la información recopilada se identificaron los aspectos que presentan conflictos u oportunidades para el municipio.

A tal fin, el desarrollo del trabajo radicó en la recopilación de información base, estudio y procesamiento de los datos existentes para la elaboración de un diagnóstico, para lo cual se acudió a fuentes primarias, secundarias y terciarias de información, con el objeto de trabajar en forma interdisciplinaria y transdisciplinaria. Asimismo, teniendo en cuenta la necesidad de 
un diagnóstico rápido y ordenado, paralelamente como forma de trabajo, se propuso la aplicación de la Metodología ICES. Los indicadores empleados constituyeron una herramienta fundamental para identificar rápidamente las problemáticas criticas actuales del municipio, que a partir de resultados obtenidos del análisis, nos permitieron definir el estado de cada uno de los temas abordados y de esta manera identificar y priorizar los puntos que requieren especial atención y formular un Plan de Acción con intervenciones óptimas.

Para poder abarcar el problema de forma ordenada se fijaron diversas escalas territoriales de trabajo para la elaboración de las actividades de relevamiento de campo. Por ello, para el Chaco y la región, se fijó la escala macro. A nivel departamental se estableció la escala mezzo y el área definida como "Chacras" es tomada a nivel microregional. A partir del tratamiento de imágenes satelitales e información cartográfica, se generaron mapas.

Para la recolección de datos y antecedentes, se plantearon como instrumentos: guías de entrevistas y planillas de relevamiento. Las tareas de campos se llevaron a cabo en equipo lo que posibilitó optimizar el tiempo de trabajo en el sitio.

El objetivo del presente trabajo consiste en "Planificar la ciudad y el territorio de Presidencia de la Plaza para lograr el desarrollo de un sistema de gestión estratégica, eficiente y consensuada que contemple tanto los aspectos sociales como económicos y ambientales, mejorando la calidad de vida de la población, promoviendo un crecimiento inclusivo y un desarrollo económico sustentable, que incluya en igualdad de oportunidades a toda la población”.

Siendo el propósito dotar a la administración pública local de un instrumento de gestión territorial para la toma de decisiones estratégica con consenso e impacto positivo en el orden económico, social y ambiental.

La propuesta busca replantear la ocupación del suelo urbano, en relación a la demanda sobre el ecosistema.

\section{CARACTERIZACIÓN DEL TERRITORIO}

El departamento Presidencia de la Plaza, surge a partir de una estación del ferrocarril de Barranqueras a Metán en 1910, estableciéndose en uno de los principales núcleos habitacionales de la provincia durante la primera mitad del siglo; y está constituido por un solo municipio, estando en la ciudad cabecera la sede del gobierno municipal. Posee una superficie de 2.284 km2, el ejido municipal tiene una superficie de 2.532 has. y su población es de 12.499 habitantes, según INDEC 2011. Se encuentra ubicado en el centro este de la provincia del Chaco, lindando al Noroeste con el departamento Sargento Cabral, al Este con el departamento Dónovan, al Oeste con el departamento 25 de Mayo y al Sur con el departamento Tapenagá. La ciudad cabecera del mismo nombre, se encuentra a los $26^{\circ} 6^{\prime}$ latitud sur y $59^{\circ} 30^{\prime}$ longitud oeste, a 73 metros sobre nivel del mar. 
En cuanto a su economía, es importante la actividad ganadera. Otro de los principales ingresos lo compone la carpintería con especies locales. Asimismo, es significativo destacar la "Fiesta Provincial del Chicharrón", que se realiza todos los años.

La Ruta Nacional No 16 constituye su principal acceso; parte de un Corredor Bioceánico que une Brasil y Chile. Desde luego, la misma la conecta al sudeste con La Verde y Resistencia, y al Noroeste con Machagai y Presidencia Roque Sáenz Peña. La Ruta Provincial No 7 corta transversalmente a la anterior y la vincula al norte con Colonias Unidas y Las Garcitas, y al sur con Charadai. Esta ruta es de tierra, aunque se está concretando su pavimentación hacia el norte.

La Ruta Nacional No 16 es considerada actualmente un estructurante de movilidad de gran relevancia para la región. Este corredor está calificado, según el Plan Estratégico Territorial de la Provincia del Chaco, con capacidad saturada o congestionada, además de contar con muchas poblaciones en crecimiento a lo largo de su trayectoria.

Existe un proyecto latente que repercutirá en la zona y es la recuperación del transporte Ferrocarril, Ramal Belgrano, el cual generará una creciente demanda de pasajeros y carga; estas nuevas intervenciones en el territorio, traen consigo una sumatoria de efectos que podrían alterar el tejido urbano y desconectar parte de las zonas urbanas inmediatas, por ello, es necesario que el Plan Estratégico contemple en su formulación los lineamientos generales de homogeneidad urbana e interrelación espacial, sana mezcla de usos del suelo y equidad de cargas y beneficios.

\section{1 | CARACTERIZACIÓN DE LA SITUACIÓN SOCIO-ECONÓMICA, TERRITORIAL Y AMBIENTAL, Y EVALUACIÓN FÍSICO-FUNCIONAL DEL SOPORTE URBANO}

\subsection{1 | Lo estructurado existente}

La Traza de la ciudad fue mensurada siguiendo un sistema de subdivisión del suelo muy regular, con una orientación a $1 / 4$ rumbo $\mathrm{NO} / \mathrm{SE}\left(26^{\circ} 6^{\prime}\right.$ latitud sur y $59^{\circ} 30^{\prime}$ longitud oeste, a 73 metros sobre nivel del mar), a partir de dos ejes cartesianos cuya intersección constituye el centro simbólico fundacional de la ciudad de Presidencia de la Plaza, que hoy configuran los terrenos del ex Tiro Federal y reservas de FFCC Argentinos. Estos dos ejes organizan el mapa funcional y mental de la ciudad, incorporando cuatro diagonales muy presentes en la identidad local.

La expansión dispersa y discontinua está estrechamente vinculada al comportamiento del mercado de suelo que, ante la presión de la demanda, eleva los precios. Este factor produce una ciudad fragmentada, que impide el acceso de la población socialmente más vulnerable del mercado formal. Paralelamente, la inversión pública en vivienda social, en el marco de un mercado de suelo no regulado, favorece la especulación inmobiliaria por parte de los propietarios de los lotes vacantes intermedios privados, que se apropian de los beneficios derivados de la extensión de las redes hacia los límites más alejados de la urbanización. Este proceso expansivo suele entrar en conflicto con los usos rurales del entorno y avanzar sobre áreas de vegetación natural o con severas restricciones ambientales. Esta situación es particularmente crítica en las áreas de 
agricultura intensiva en el periurbano de las grandes ciudades o en oasis de riego. En cuanto a las restricciones ambientales, hay ejemplos de expansión en tierras con riesgo de deslizamientos o en áreas pasibles de padecer inundaciones. Esta cuestión genera dos situaciones, una es la dispersión de los proyectos de vivienda y por otro lado la distribución anárquica de los parcelamientos respecto de la trama urbana que le da origen al pueblo (damero tradicional).

El municipio Presidencia de la Plaza, se organiza en base a una trama cuadrada a manera de "damero urbano", que resulta de la manera como están dispuestos entre si las formas construidas y los espacios públicos. El damero patrón se manifiesta como una trama urbana más legible y aprehensible, y en la medida que el municipio se desarrolla a través de las nuevas intervenciones de asentamientos humanos, traen consigo problemas de circulación, y, por tanto, de movilidad dado que lo hacen de manera anárquica, fragmentada y sectorial.

El tejido urbano, en cambio, se caracteriza por su homogeneidad en la poca altura de sus edificaciones entre tres y nueve metros, lo cual otorga condiciones ambientales favorables -cuota de luz natural, asoleamiento, ventilación, higrometricidad, privacidad, ruidos, y visuales exteriores que tienen los ambientes habitables-.

Es legible la situación de interrupción de la trama y del tejido urbano en relación a los nuevos parcelamiento tanto provenientes de proyectos no solo del Instituto de Vivienda sino de varios actores, sin una coordinación del municipio y sin una idea clara y pensada a futuro. Se aprecia claramente los barrios que impactan negativamente respecto de la estructura visual del conjunto.

Desde luego, al referirnos a la trama se contemplan dos factores fundamentales que la componen: la calle y la parcela. Por un lado, se considera el trazado, con referencia a la estructura viaria que divide el territorio en manzanas, y por el otro, el tejido parcelario como una subdivisión del territorio con respecto a la manzana.

La discontinuidad en el trazado de la trama urbana es el resultado del crecimiento del municipio, provocando un quiebre, una ruptura de la estructura viaria. La ruptura de la trama en su repetición de discontinuidad del trazado, generan un límite entre sectores próximos que poseen el mismo uso del suelo, como es en este caso el uso residencial, lo que dificulta la integración urbana, quedando estos límites bien marcados.

Como punto provechoso se rescata los grandes lotes factibles de nuevos parcelamientos. Es preciso y posible a la vez, implementar nuevas políticas de gestión sobre la fracción de la ciudad, con el objeto de lograr la continuidad de las calles, más en esencia, la continuidad de la trama urbana.

Asimismo, como producto de acciones inapropiadas, existen preocupaciones en cuanto a:

- Inundaciones en ciertas zonas por la baja pendiente del relieve.

- Escasez de espacios verdes públicos y ausencia de reservas ambientales.

- Carencia de planificación y ordenamiento del ejido urbano.

- Uso irracional de los recursos.

- Deterioro e insuficiencia de la infraestructura existente y sin visión de futuro.

- Dispersión urbana, generando problemas de insostenibilidad ambiental y económica. 
Por otro lado, el desarrollo acelerado de la población, genera un crecimiento y, por tanto, mayor consumo de las redes de servicios como así también de equipamientos, movilidad, de espacios verdes públicos. No obstante, el municipio se caracteriza por la insuficiencia en redes de transportes, escasez de áreas verdes accesibles y reservas de protección ambiental. Asimismo, este incremento también significa mayor cantidad de generación de residuos sólidos urbanos.

\subsubsection{Contexto actual del crecimiento urbano del Municipio}

El crecimiento de las ciudades es un fenómeno urbano, que se caracteriza por utilizar patrones de usos del suelo complejos, debido a la diversidad de actividades humanas, lo cual conlleva a un desarrollo poco ordenado. En este sentido resolver las irregularidades y estructura interna de las ciudades adquiere un rol esencial.

Las tendencias de urbanización y expansión urbana se apoyan a la baja densidad y a la segregación espacial de los usos de suelo. Es importante analizarlo desde sus variables morfológicas, económicas, sociales ambientales e incluso políticas.

Puntualizando en el crecimiento urbano de Presidencia de la Plaza, en el ámbito local, carece de una aplicación de una política o norma respecto a la asignación del uso del suelo. En este sentido, una de las actividades iniciales fue el diagnóstico expeditivo del Municipio, donde se analizaron las principales características de la zonificación. Por otro lado, se realizó un estudio integrado con aspectos del entorno urbano y ambiental, implementando una metodología de relevamiento in situ para caracterizar desde el punto de vista socio-ambiental el microcentro.

\subsubsection{Movilidad, conectividad y accesibilidad}

Presidencia de la Plaza se conecta con las provincias del Norte a través de la Ruta Nacional No 16 en dirección E-O -la conecta al NO con Machagai y Presidencia Roque Sáenz Peña, y al SE con La Verde y Resistencia-.

Este corredor estructurante une las provincias de Corrientes, Santiago del Estero, Salta y Chaco respectivamente. Se encuentra totalmente asfaltada y su extensión es de 707 km., con $32 \mathrm{~km}$. de autovía en el tramo Resistencia-Puerto Bastiani, y durante su paso por Presidencia Roque Sáenz Peña. Se encuentra en proceso de construcción 27 km. más de autovía entre Makallé y Puerto Bastiani. Asimismo, el cruce sobre el Rio Paraná se efectúa por el Puente General Manuel Belgrano, el cual fue la primera vinculación terrestre entre la Mesopotamia y el resto del país.

La Ruta Provincial No 7 la corta transversalmente, vinculándola al norte con Colonias Unidas y Las Garcitas, y al sur con Charadai. Esta ruta es de tierra, no obstante, se está llevando a cabo su pavimentación a lo largo de $38 \mathrm{~km}$. entre la localidad de Presidencia de la Plaza y Colonias Unidas, al noroeste de Resistencia, que hoy es de tierra.

En materia ferroviaria, la provincia de Chaco cuenta con $1.221 \mathrm{~km}$. de vías del Ferrocarril General Belgrano. No obstante, esta infraestructura se encuentra clausurada, desmantelada y abandonada. 
En términos urbanos, la inactividad del Ramal F17 tiene consecuencias negativas para el desarrollo de la provincia, ya que priva a las ciudades de un medio de transporte como así también conforman importantes barreras y áreas baldías que impactan negativamente sobre su desarrollo.

Su línea principal parte desde la estación cabecera de Retiro, en la Ciudad de Buenos Aires, y se dirige hacia el norte argentino, recorriendo las provincias de Buenos Aires, Santa Fe, Córdoba, San Luis, Mendoza, San Juan, La Rioja, Catamarca, Tucumán, Santiago del Estero, Formosa, Salta, Jujuy y Chaco respectivamente. Cumple un rol económico clave para la producción de las provincias del noroeste y noreste del país, puesto que permite transportarla hacia los principales centros de consumo y puertos.

A nivel municipal, Presidencia de la Plaza se encuentra estructurada por la R. N. $\mathrm{N}^{0} 16$, de doble mano y en proyecto se encuentra la autovía que une Resistencia con Presidencia Roque Sáenz Peña con colectora lateral de tierra a ambos lados, que permite desplazamientos NO-SE -eje de gran demanda de flujos de bienes y personas-.

A la misma se conectan cuatro ejes viales de menor importancia pero de gran flujo en sentido NE-SO con capacidad para vehículos livianos y pesados -calle Martin Farías, Bernardo Pérez, Maipú y Alberdi-, generando a su vez accesos, que si bien tienen una gran jerarquía, no son apreciables desde la ruta a nivel vehicular.

Dada la cercanía de estos ingresos, se percibe una clara dispersión de los mismos sumados al acceso provincial $\mathrm{N}^{\mathrm{O}} 7 \mathrm{y}$ la ruta departamental.

Desde luego, en términos urbanos, se observa la carencia de un destino claro, de un centro estructurante. Es decir, no se materializa un núcleo funcional.

\subsection{4 | Red vial}

La configuración de una red vial coherente, completa y abierta es un aspecto fundamental de las propuestas y reglamentaciones, de ella depende la efectividad funcional del área urbana y se constituye en la columna vertebral de su estructuración, por lo cual las arterias que las forman debe estar calificada en cuanto al rol funcional que debe cumplir, ancho de vía necesario y control de las actividades que puedan localizarse sobre las mismas.

Se identifican cinco categorías de vías circulatorias:

- Red vial primaria

"Está constituida por los tramos de rutas nacionales y provinciales, que posibilitan los accesos regionales y distribuyen el tránsito desde los mismos hacia la red vial secundaria" (Código de Planeamiento Urbano de la ciudad de Resistencia).

- Red vial secundaria

"Está constituida por las arterias de tránsito rápido que distribuyen los flujos principales de circulación entre los distintos sectores del complejo urbano y desde los mismos hacia las salidas regionales. Por su naturaleza estas arterias deben disponer de un ancho de vía 
suficiente y ser periféricas a las áreas centrales y de mayor densificación con el fin de evitar la congestión de las mismas.

Al mismo tiempo por su ubicación dentro de la red, algunas están destinadas preferentemente al tránsito de cargas pesada, otras al de cargas liviana y otras al de tránsito liviano" (Código de Planeamiento Urbano de la ciudad de Resistencia).

- Red vial terciaria

"Está constituida por las vías que interconectan entre sí las áreas centrales y comerciales y canalizan el mayor flujo urbano por lo cual la velocidad de circulación se torna más lenta, esta red configura una trama cruzada con la red secundaria de modo que desde cualquier punto de la misma es posible alcanzar, a corta distancia una vía rápida de distribución”. (Código de Planeamiento Urbano de la ciudad de Resistencia).

"Está representada por las múltiples calles de acceso a cada parcela urbana por lo cual no integran la categoría de red principal. No obstante, deben guardar una red terciaria y es admisible su intersección con la red secundaria, pero de ningún modo puede tolerarse su vinculación directa con la red primaria para la cual deben preverse calles laterales de servicio".

"Cada categoría de vías forma una red continua entrecruzada con la categoría superior e inferior por lo cual es posible desplazarse sin discontinuidades dentro de cada sistema, así como de un sistema a otro, desde cualquier sector del complejo urbano total." (Código de Planeamiento Urbano de la ciudad de Resistencia).

- Red peatonal: veredas

Son de carácter público, destinadas al tránsito peatonal. Se destaca en el municipio los espacios peatonales rotos como así también un enorme número de faltantes de veredas. Son muy pocos los frentistas que cumplen con los requisitos mínimos para la transitabilidad universal.

Según el artículo 158 de la ordenanza 10.681 (Código de Edificación de la ciudad de Resistencia), "todo terreno edificado o no, con frente a la vía pública, debe tener vereda construida" y en ese sentido, seguidamente, la normativa detalla cómo debe ser el diseño de la acera, que tendrá que extenderse en todo el largo del frente de la línea municipal hasta el cordón de la calzada.

\subsection{5 | Redes de infraestructuras y servicios}

Uno de los aspectos fundamentales del estudio de un territorio, es el análisis de las actividades, servicios e infraestructura que sirven a la población y de su área de influencia.

Por ello, si definimos al sistema urbano como un conjunto de actividades y las relaciones que esta mantiene entre sí, la estructura espacial del sistema urbano se reflejará a través de la localización y distribución de las actividades en el espacio urbano y de la dimensión espacial que adquieran sus relaciones, materializando así a la estructura física de la ciudad.

La ciudad, constituye, por tanto, un fenómeno complejo donde se concentran múltiples actividades que para su desarrollo y localización requieren la existencia de infraestructura y 
servicios, que hacen posible el desarrollo de las actividades y que a su vez las condiciona.

Presidencia de la Plaza se encuentra emplazada en un territorio con hidrografía dificultosa y el desarrollo acelerado de la urbanización desestima las condiciones de la geografía, y como consecuencia algunos sectores presentan cuestiones que inciden negativamente.

La urbanización al estar limitada por arroyos y zonas inundables, acontece problemas críticos en sectores que afectan las condiciones de vida de los residentes y el escaso desnivel perjudica el drenaje o escurrimiento natural, haciendo necesario la presencia de grandes reservorios, tanto naturales como artificiales.

\subsubsection{Sistema fluvial: zonas de riesgo}

Dentro de la estructura conceptual de la ciudad como del ciudadano, no aparece el Arroyo Plaza como bien identitario; y, como sistema y elemento que estructura el suelo, tampoco está presente en los funcionarios. Al ser un antiguo brazo y no contar con corrientes, pasar por terrenos privados y públicos, el pueblo no le supo otorgar una función, sin embargo, tiene una riqueza paisajística increíble y única a potenciar. Funcionalmente puede servir como reservorio natural, para lo cual es imprescindible dragarlo, limpiarlo. O sea, tiene un rol utilitario y un rol paisajístico, pero vitalmente, es necesario entenderlo como parte de la estructura urbana y como soporte natural; cuidarlo, mantenerlo, pensando en su espacio natural de oscilación, fluctuación propia de los humedales.

La Carta Orgánica Municipal de la ciudad de Resistencia, en lo referido al desarrollo territorial y el medio ambiente, definió el marco legal para el desarrollo de la ciudad, estableciendo la importancia de la ciencia y tecnología como instrumentos adecuados para la promoción humana, el desarrollo sostenible y el mejoramiento de la calidad de vida. Con esta propuesta normativa se pretende regular el manejo de las aguas de origen pluvial de manera que generen condiciones de impacto cero en la capacidad y funcionamiento del sistema de desagües pluviales existentes.

Los peligros de inundación en áreas urbanas son el resultado de sistemas de drenajes inadecuados o inexistentes y de la ocupación de zonas ribereñas expuestas a un alto riesgo de inundación.

\subsection{7 | Usos del suelo}

Las áreas verdes neutralizan las agresiones típicas de la ciudad; son espacios urbanos ocupados predominantemente por árboles, arbustos y otros tipos de vegetación. Presidencia de la Plaza tiene la particularidad de contar con espacios verdes en terrenos privados que se suman a los públicos para brindar una cantidad más que saludable de áreas verdes.

La Organización Mundial de la Salud recomienda que las ciudades proporcionen como mínimo $12 \mathrm{~m} 2$ de área verde por habitante. Sumando la superficie de áreas verdes públicas y privadas, Presidencia de la Plaza tiene disponible mucho más de lo recomendado por la OMS, pero carece de infraestructura necesaria para su uso, lo cual la sitúa por debajo de lo que establece la OMS. 
El racionamiento de espacios públicos merita un particular interés considerando la nueva población futura, para cumplir con los parámetros convenientes, tanto en las áreas céntricas como en las otras áreas semiurbanas del municipio.

Presidencia de la Plaza no solo tiene una cantidad de verde, sino una buena calidad de verde. La vegetación del municipio es antigua y nativa, lo cual mejora su capacidad de absorber $\mathrm{CO} 2$ y servir de "pulmón verde".

Las áreas verdes urbanas ayudan a regular el microclima y el ruido de la ciudad, mejoran la calidad del aire, disminuyen la erosión del suelo y sirven de hábitat y alimento para la fauna silvestre. Asimismo, reducen los niveles de anhídrido carbónico (CO2), monóxido de carbono (CO) y anhídrido sulfúrico ( $\mathrm{SO} 2$ ), principales contaminantes del aire de las ciudades, y contribuyen a la formación de microclimas urbanos placenteros. Se estima que el ser humano consume entre 500 y 600 gramos de óxido al día, un solo árbol adulto y frondoso puede producir esa cantidad en poco más de 20 minutos.

\subsection{8 | Suelo vacante}

El suelo vacante -suelo urbanizado sin destino concreto y usualmente sin mejoras edilicias o en desuso- son suelos que de una u otra manera están sub-urbanizados y, en el caso de suelo de dominio privado, un gran porcentaje se encuentra a la espera de valorización dentro del mercado inmobiliario. Si bien la oferta de suelo es necesaria para satisfacer la demanda y el equilibrio de los precios, los procesos especulativos de retención de suelo tergiversan en oferta contribuyendo al aumento generalizado del valor del bien. Los actuales procesos de urbanización que, en muchos casos, no responden a las lógicas de continuidad y completamiento a la trama urbana sino que conllevan un proceso de discontinuidad tanto espacial como temporal, contribuyen a generar estos suelos vacantes.

El suelo vacante se divide en dos categorías dependiendo de su propiedad:

- Propiedad Pública

El ferrocarril como Tierras Nacionales Vacantes -Ex Campos Tiro Federal, 9Has.-: El predio Estación y la traza de vías con características particulares históricas se configura hoy como un área de vacancia en espera de nueva configuración y uso urbano.

- Propiedad Privada

Las manzanas y los predios privados se distribuyen en toda la planta urbana, con tendencia a concentrar su ubicación en los bordes periféricos.

\section{2 | EJES PROBLEMÁTICOS}

A partir del diagnóstico se presentan una serie de problemas a mejorar o áreas a potenciar mediante su desarrollo, que se han agrupado según distintas temáticas. Surgen así modos 
de acción que proponen detectar las causas de la situación actual que generan algún problema o incompatibilidad en el día a día de sus habitantes para poder identificar los objetivos necesarios que permitan desarrollar programas y proyectos para lograr el tipo de ciudad deseada.

Se enmarcan a continuación los principales ejes problemáticos establecidos para el municipio de Presidencia de la Plaza: Urbano-Ambiental-Legal y Socio-Económico.

El eje Urbano-Ambiental se estructuró en base a indicadores físicos -servicios, infraestructura, equipamientos, accesibilidad, legal e institucional, mientras que el eje socio-económico, se planteó de modo más general.

Sobre esta base se ha construido una matriz de identificación de las problemáticas y potencialidades relevantes que permite establecer ejes direccionales, facilitando la toma de decisiones:

Aspectos Aspecto Urbano-Ambiental-Legal
Poca vinculación entre los municipios vecinos.
Carencia de adecuación y completamiento de
la estructura de gobierno municipal a las nue-
vas demandas.
Carencia de equipo técnico para lograr mayor
eficiencia.

Institucional Falencias en los equipamientos del edificio Capacidad de gestión para articular con el gomunicipal, principalmente falta de informati- bierno nacional y provincial la ejecución de zación en todas las áreas. planes, programas y proyectos.

Carencia de actualización del área catastro (falta digitalizar el catastro, incorporar recursos humanos, actualización de la metodología de trabajo).

Se desconoce registro y sistematización de normativas legales, leyes, decretos.

Legal Carencia de una normativa integral que regule un registro integral del aspecto normativo legal. los usos y las formas de ocupación de suelo urbano, y que oriente la expansión de la localidad.

Importante déficit en obras de infraestructuras urbanas, en algunos casos no existe (red Buen funcionamiento de los servicios $-85 \%$ de de gas natural) y en otros requiere completa- la ciudad posee red de agua potable y energía miento y mejora de servicios (agua potable, eléctrica-. energía eléctrica, cloaca).

Servicios, Infraestructuras Problemas derivados de la carencia de des- tribuirían a minimizar los efectos de los excey equipamientos agües pluviales, crecimiento de la población sos de agua por lluvia. en zonas no aptas -anegadas- por lluvias.

Bajo porcentaje de redes pavimentadas, los Viviendas. accesos desde Ruta Nacional se identificaron como gran cantidad y por tanto, poco claros y definidos. 


$\begin{array}{lll}\text { Aspectos } & \begin{array}{l}\text { Problemas } \\ \text { Deficiencias en el mantenimiento de espacios } \\ \text { peatonales. }\end{array} & \begin{array}{l}\text { Potencialidades } \\ \text { Buena distribución y estado de caminos natu- } \\ \text { rales. }\end{array} \\ \begin{array}{ll}\text { Servicios, } \\ \text { Infraestructuras }\end{array} & \begin{array}{l}\text { Deficiencias en el mantenimiento de los equi- } \\ \text { Poca población viviendo en situación de ocu- }\end{array}\end{array}$

y equipamientos pamientos sociales y recreativos públicos. pación irregular de la tierra.

Deficiencias en las condiciones edilicias, insu- Estratégica comunicación de la ciudad con la remos y recursos humanos. gión a través de las rutas nacional y provincial.

Incompleta gestión de residuos sólidos urbanos. Proyecto de completamiento de una planta de tratamiento.

Poco desarrollo urbano de las áreas aledañas a Se pondera el emplazamiento del municipio la antigua zona de las vías del ferrocarril. en un punto estratégico a escala regional, con importantes escenarios y recursos naturales

Baja calidad y escasa superficie de espacios de gran valor. verdes públicos dedicado a la recreación.

Disponibilidad de predios intersticiales que Existencia de barrera física -Arroyo Plaza- que podían incorporarse al sistema de espacios

Ambiental condiciona la extensión de la cuadrícula. públicos.

Carencia de planificación urbana.

Posibilidad de generar urbanizaciones compatibles con la naturaleza regional, con sitios de Aplicación de sistema tradicional en desuso recreación y atractivo para el turismo. para el tratamiento de líquidos cloacales, dado el grado de contaminación que produce. Se dispone de sistemas nuevos para el trataProximidad de la laguna de oxidación de líqui- miento de líquidos cloacales que reduce la dos cloacales al ejido urbano. contaminación ambiental.

\section{Aspecto Socio-Económico}

Altos índices de NBI.

Social

\section{Carencia de oferta de educación universitaria conciencia en la}

pública (diseño de muebles, diseño industrial,

ingeniería forestal, agronomía, etc.) en áreas Conciencia en generar o promover capacitatécnicas y tecnológicas respecto del perfil pro- ción en gestión empresarial y comercial. ductivo y económico del territorio.

Calidad de la producción primaria local, especialmente agrícola.

Proximidad del Parque Nacional Chaco como potencial turístico.

Económico

No existen actividades secundarias integradas Se destaca al recurso natural como uno de los a la producción primaria local. recursos claves para el desarrollo del sector.

Altos índices de NBI.

Fácil comunicación vial a través de las rutas nacional y provincial.

Se destaca la actividad ganadera como principal actividad económica.

Creciente demanda de alimentos. 


\section{3 | SÍNTESIS MODELO INICIAL}

En la primera fase de este informe, la recopilación de información provino de diferentes fuentes que, luego de ser procesada y ordenada, decantó en un proceso de análisis hasta llegar a la elaboración de un diagnóstico.

Concluida la fase de diagnóstico, se cuenta con una primera visión de los temas más críticos y favorables para el municipio. Algunos de ellos, para ser analizados y evaluados correctamente y contar con mayores elementos para la definición de los programas y proyectos prioritarios del Plan de Acción.

El Modelo Inicial sintetiza los principales componentes que estructuran el territorio a partir de la integración del conocimiento alcanzado a través del análisis de sus dimensiones -Dimensión Urbana, Socio-Económica, Ambiental y Legal-. El mapa síntesis representa en sí, la organización espacial y el funcionamiento del municipio con sus respectivos conflictos y oportunidades.

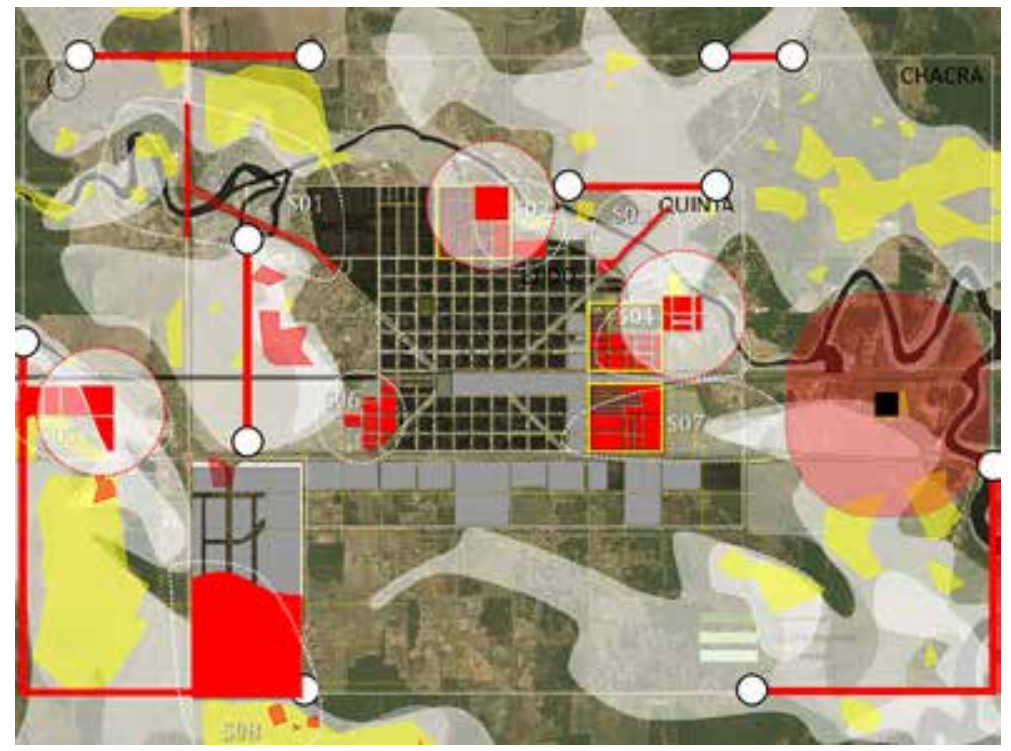

Ilustración 01: Modelo inicial Municipio de Presidencia de la Plaza.

Fuente: Elaboración propia.

\section{SÍNTESIS MODELO DESEADO}

Surge como una respuesta superadora del Modelo Inicial y representa la imagen objetivo de la estructura territorial que se propone construir mediante la implementación del plan. En esta instancia se proponen políticas públicas especificas en función de las problemáticas y potencialidades detectadas en la fase de diagnóstico y considerando los diferentes escenarios posibles en el futuro. 


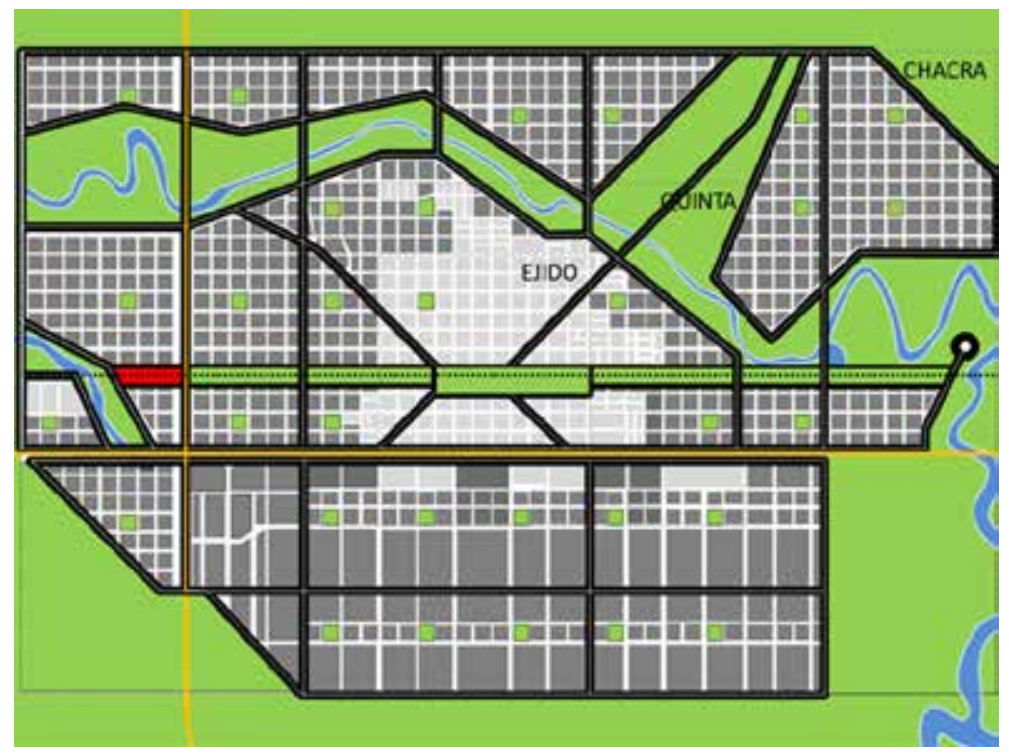

llustración 02: Modelo deseado Municipio de Presidencia de la Plaza.

Fuente: Elaboración propia.

Presidencia de la Plaza se posiciona como centro agro productivo de la Región Centro Chaqueña. Se distingue como municipio saludable, ordenado, sustentable y socialmente integral, y se caracteriza por sus grandes extensiones naturales y bienes paisajísticos.

\section{1 | DEFINICIÓN DE ESCENARIOS}

La utilización de la prospectiva como herramienta permite anticipar diferentes escenarios posibles para ver las oportunidades y gestionar los riesgos futuros desde una posición de ventaja, conectando las acciones de corto plazo con la perspectiva de largo plazo.

\subsection{1 | Propuesta: Nueva Centralidad}

Renovación del área central actual del municipio, ubicada en torno al predio Ex Tiro Federal, y a su vez mejorar los principales accesos, calles internas y principales caminos rurales, ya que se encuentran en mal estado y perjudican la movilidad de la población.

Esta propuesta implica la incorporación de nuevos espacios públicos de nexo entre el Norte y el Sur del municipio, a partir de la progresiva reconversión urbanística del área ferroviaria, sitio clave para el desarrollo e integración del Municipio de Presidencia de la Plaza.

\subsection{2 | Propuesta: Recuperación del predio Ex Tiro Federal -Predios Urbanos Disponibles Subutilizados-}

El predio de FF.CC. es una interface entre la zona urbana residencial y comercial. Este predio es una gran pertenencia y oportunidad como espacio verde recreativo y de esparcimiento 
para el municipio, que posibilitará lograr una mejor integración de la ciudad. Los espacios vacíos existentes entre la mancha urbana actual y los límites de la ciudad, corresponden a privados.

Se lo ve como un potencial integrador de toda la ciudad a lo largo de su extensión generando una avenida estructurante cuyo espacio central (entre vías de circulación) el espacio debe ser de uso recreativo, esparcimiento con tendencia a fomentar y potenciar la comunicación dado que estratégicamente se encuentran las vías del ferrocarril, apeaderos, pudiendo instalarse playas de transferencia de carga y puntos de contactos ferro-automotor que potencien a la ciudad y su perfil agro ganadero y exportador.

\subsubsection{Propuesta: Nuevos Amanzanamientos y Parcelamientos}

Uso de la manzana en cuadricula como una fase formal y organizativa

La principal eficacia de la cuadricula es su simplicidad; la organización racional de las calles en una red geométrica simplifica el complejo proceso de construcción de la ciudad y permite a su vez, la urbanización de áreas extensas con una sola identidad y una imagen coherente. Asimismo, ofrece una ciudad funcional, legible y optimiza la infraestructura.

En el proyecto de urbanismo, el uso de la cuadricula obtiene una distinción muy clara del espacio público y privado.

En la ilustración o7 vemos la prospectiva desde la cuadricula inicial, la cuadricula actual y por último la cuadricula deseada. Pasamos desde un modelo inicial ordenado, un modelo actual anárquico y el modelo propuesto planificado, donde hay jerarquías respecto de las vías circulatorias de manera de generar una estructura de identificación.

El Instituto de Vivienda, a través de sus distintos planes, incide fuertemente en la zonificación y uso del suelo, generando por un lado un saneamiento en la demanda habitación, y por otro lado impactos negativos, desequilibrando áreas como resultado de una política anárquica.

En la ilustración o8 se muestran dos propuestas de amanzanamiento de densidad baja y dos propuestas de amanzanamiento de densidad media, donde diseño y la cantidad de parcelas es solo indicativo. Es una propuesta que puede variar de dimensiones pero que, desde luego, se respeta los ejes, anchos y manzanas existentes.

El tamaño de la manzana deberá respetar la trama original de Presidencia de la Plaza.

Se recomienda que los pasajes que cortan la manzana original no tengan una continuidad mayor a dos cuadras, incluso deberían ser discontinuos, de tal manera de no generar estructuras identitarias paralelas que desorienten al ciudadano.

\subsection{3 | Propuesta: Espacios verdes}

Planificar el crecimiento del tejido urbano de la ciudad de manera equilibrada entre la masa edificada y los espacios libres, mejora la calidad de vida urbana resaltando las potencialidades paisajísticas y ambientales del municipio. Accesible a los ciudadanos para la recreación. 
El avance de la urbanización del municipio impacta en sus condiciones ambientales, por ello resulta fundamental proteger, conservar e incrementar las áreas verdes urbanas, ya que desempeñan funciones esenciales para la calidad de vida de los habitantes. El cuidado, manejo y mantenimiento de los espacios verdes requiere del compromiso tanto de la sociedad como de las entidades gubernamentales vinculadas con ellas.

Por ello, impulsar el desarrollo de un sistema de áreas verdes, es otro de los temas prioritarios a considerar en el proyecto.

Como se dijo anteriormente, las áreas verdes y espacios abiertos desempeñan un conjunto de funciones esenciales en el bienestar y en la calidad de vida de los ciudadanos. No obstante, los innumerables beneficios ambientales de las áreas verdes, cabe mencionar la dimensión social como una de las más relevantes para ciudad. Como equipamiento social, las áreas verdes son el soporte en el esparcimiento y la recreación, pues constituyen espacios privilegiados en la reproducción y el reforzamiento de la identidad del núcleo urbano.

Por esta razón, en la propuesta urbana se destina una superficie del 12\% de áreas verdes que corresponde, según su criterio, al mínimo exigible para una razonable urbanización.

La desigual distribución de áreas verdes públicas para la recreación trae como consecuencia que los habitantes tengan que buscar áreas verdes adecuadas para su esparcimiento, por lo que restringe sus visitas, como así también su mantenimiento.

$\mathrm{Su}$ implementación seria convertirlas solamente en un espacio residual con muy poco impacto urbano. Lo correcto es que las áreas verdes estén concentradas y no distribuidas en superficies dispersas, deben estar accesibles a la población, de una manera que puedan disfrutar de sus beneficios con la obligación de conservarlos y cuidarlos.

Los espacios verdes públicos deben tener las siguientes premisas:

- Ser el 12\% de la superficie total a intervenir,

- beben contener infraestructura acorde para el uso recreativo,

- el $12 \%$ destinado a espacio público debe estar en zona no anegable y exenta de circulación pública de manera que sea superficie total neta para el óseo, recreación y uso público en general, independientemente de las circulaciones de vinculación.

\subsubsection{Propuesta: Crecimiento urbano - zonificación}

Se proyecta un crecimiento del municipio priorizando la plena ocupación, consolidación y cualificando la planta urbana existente a partir del mejoramiento y completamiento de las infraestructuras y la provisión de equipamientos.

Es imprescindible promover una política de gestión urbana de modelo compacto por sobre los modelos expansivos que originan ciudades de baja densidad.

La planta urbana actual de Presidencia de la Plaza, aun presenta una forma compacta y unitaria. Por ello, es necesario generar instrumentos normativos y de proyecto que supervisen el proceso de incorporación de tierra rural a urbana, clasificando el suelo para el planeamien- 
to, orientando determinados crecimientos de la planta y exigiendo condiciones urbanísticas a las nuevas operaciones de extensión urbana.

Reformulación de dos sectores de la trama urbana. Uno del Instituto de Vivienda por la cercanía del curso de agua y la definición de la línea de ribera, incluso está en zona inundable. Asimismo, se pretende la relocalización de la laguna de oxidación, dada su proximidad a la línea urbana y pasando la línea de chacra, y de este modo, recuperar 25 chacras para suelo urbano.

El crecimiento hacia el sector oeste deberá preverse un saneamiento de la zona inundable, contemplando el Código de Aguas y generado una compensación, relocalizando los volúmenes de aguas recuperados como suelo urbano.

\subsection{5 | Propuesta: Supermanzanas}

El objetivo fundamental de esta propuesta es la ordenación territorial y desde luego, promover la participación ciudadana en las decisiones que se adopten en materia de reorganización territorial.

La supermanzana es una nueva célula urbana forma de organización urbana que, con su implantación, aporta soluciones ligadas a la mejorar de la disponibilidad y calidad del espacio público.

\subsubsection{Propuesta: Accesibilidad en el espacio público}

Se propone la recuperación y mejoramiento de las calles, pavimentando de acuerdo al tránsito y la categoría con un perfil vial que incluya la calle, veredas y bici sendas, señalética y árboles de las calles y avenidas estructurantes del municipio. Asimismo, se pretende un plan de ciclo vías que promueva el uso de la bicicleta como un medio de transporte primordial con seguridad vial.

El espacio público la conforma la dimensión colectiva de la ciudad, por tanto, es el lugar de la representación e identificación colectivas.

La apropiación de este espacio por parte de todos los integrantes de la sociedad es parte del derecho a la ciudad, y por ello se deben favorecer usos y actividades que permitan acceder al mismo. La implementación de criterios de accesibilidad en el espacio público es una medida que beneficia al conjunto de la población, no solo a las personas con discapacidad.

Los elementos a considerar en el diseño son:

- Pavimento y solados: Tipo y estado de conservación.

- Desniveles existentes: Diseño universal.

- Cruces peatonales de calles: Localización, situación de desnivel entre la vereda y la calzada, factibilidad.

- Señalización: Localización, tipo, existencia de semáforos y señales sonoras.

- Estacionamientos: Localización, dimensiones.

- Iluminación: Localización alumbrado público, tipo de artefactos. 
El proyecto de pavimento requiere de perfiles alternativos acordes al drenaje o escurrimiento de las aguas, articulándolo con la cota mínima de umbral de los domicilios, lo que genera a su vez entre lo que es la calle pública y la vereda de dominio privado y uso público, la falta de control originando obstáculos en la senda peatonal.

La vereda debería permitir el libre paso, evitando interrumpir así la circulación peatonal o su uso, pensando en la accesibilidad universal. Las mismas contemplarán un ancho mínimo de 2,00 m. Esta medida responde a garantizar el paso de dos personas, una de ellas en sillas de ruedas.

En casos donde la topografía del terreno genere una diferencia de nivel entre una acera y otra existente, la transición se resolverá mediante un plano inclinado, con una pendiente máxima de $8 \%$.

La composición del solado será de forma tal que sean estables, antideslizantes en seco y en mojado, con el fin de brindar una superficie para circulación peatonal con seguridad.

Por ello, los proyectos de calles deberán contemplar además la ejecución de veredas, resolviendo correctamente los puntos de encuentro.

En el listado se da un orden de actuación de trabajos pensando en una solución integral al problema.

En cuanto a los estacionamientos, estarán demarcados y señalizados con el símbolo internacional de accesibilidad aprobado por la Norma IRAM 372, pintado en el solado y también colocado en señal vertical a una altura tal que pueda ser visto mientras un vehículo es estacionado.

Otro elemento a considerar en el proyecto, es la iluminación en calles. Es primordial ofrecer un grado aceptable de uniformidad en la iluminación como así también facilitar el mantenimiento, satisfacer las condiciones de estética y dar un grado óptimo de confiabilidad.

Para favorecer la orientación de las personas, el recorrido será señalizado en el solado mediante el empleo de franjas-guías y franjas de prevención que orienten al mismo. Las primeras serán baldosas con bandas direccionales en recorridos lineales, cuyas acanaladuras estarán orientadas en la dirección de la marcha. Mientras que las franjas de prevención serán señalizaciones en solados, compuestas por baldosas con botones de color amarillo.

\section{REFERENCIAS BIBLIOGRÁFICAS}

Ministerio de Planificación Federal, Inversión Pública y Servicios 1816 - 2016. Argentina del Bicentenario Plan Estratégico Territorial. Avance 2008.

Ministerio de Planificación Federal, Inversión Pública y Servicios 1816 - 2016. Argentina del Bicentenario. 
Ministerio de Planificación Federal, Inversión Pública y Servicios, "Plan Estratégico Territorial Avance II: Planificación Estratégica Territorial”.

Ministerio de Planificación Federal, Inversión Pública y Servicios, “Plan Estratégico Territorial Avance II: territorio e infraestructura”.

Ministerio de Planificación Federal, Inversión Pública y Servicios, “Plan Estratégico Territorial Avance II: Argentina urbana".

Ministerio de Planificación Federal, Inversión Pública y Servicios, "Plan Estratégico Territorial Avance II: Integración Territorial Internacional”.

Cámara de Diputados de la Provincia del Chaco. Ley 4088. Áreas de influencia de los Municipios de la Provincia, sus límites y linderos.

Plan Estratégico territorial de la Provincia del Chaco (2013). Tomo 1, 2, 3, 4. Programa de fortalecimiento institucional de la subsecretaría de planificación territorial de la inversión pública.

Plan Estratégico Garupá -PEG-. Ordenanza No 385.

PE -Plan Estratégico Malabrigo-. Plan Estratégico para el Ordenamiento Urbano y el Desarrollo Territorial del Municipio de Malabrigo 2916-2030.

Plan Estratégico Territorial Coronel Du Graty, Provincia de Chaco.

BID (2009). Plan Chaco Explora Plan Estratégico de Turismo Sustentable. Por un turismo serio y de calidad. Noviembre Gobierno del Pueblo de la Provincia del Chaco. Subsecretaría de Turismo. ESTUDIO 1.EG.133 "PLAN ESTRATEGICO DE TURISMO SUSTENTABLE DE LA PROVINCIA DEL CHACO”.

BID (2013). Guía metodológica. Anexo 2 Indicadores de la Iniciativa Ciudades Emer-gentes y Sostenibles.

BID (2014). Guía metodológica Iniciativa Ciudades Emergentes y Sostenibles. Segunda edición. 
BID (2015) ANEXO GUÍA METODOLÓGICA. Extensión a nuevas áreas: Fomento de la competitividad y el desarrollo económico local en las ciudades intermedias de LAC. Primera Versión. INICIATIVA CIUDADES EMERGENTES Y SOSTENIBLES. SEPTIEMBRE

BID (2015). Guía Metodológica. Iniciativa ciudades emergentes y sostenibles. Tercera edición.

Geografía del Chaco. Amelia F. de Mahave, Adela de Sáez y Graciana de Fernández Rey.

Estructura Funcional de la Provincia del Chaco. Brian Alejandro Thomson.

Plan Hídrico Ambiental. Dr. Cesar Horacio Dellamea, Ing. Raquel Pérez y Arq. Alicia Mastandrea.

Normativas Ambientales de la Provincia del Chaco. Tomo II. Tec. Universitario Ambiental - José Elías Memendi.

\section{CURRICULUM VITAE}

\section{Alberto Patricio Mahave}

Mgter. en Arquitectura (UNL). Arquitecto (UNNE). Jefe de trabajos prácticos - Arquitectura 4 UPA, UNNE. Profesor adjunto - Morfología II, UNNE. Gestión Institucional CYT: Gerente deárea. albertomahave@gmail.com

\section{Moira Carrió}

Licenciada en Economía (UNNE).

Especialista en Docencia Universitaria (UNNE).

Profesor Titular Principios de Economía (UNNE). Profesora Adjunta a Cargo: Cuentas Nacionales y Estructura Económica Argentina (UNNE).

mcarrio@comunidad.unne.edu.ar

\section{Natalia Albendaño}

Estudiante. Facultad de Arquitectura y Urbanismo (UNNE). natalia_a.alb@hotmail.com 